POS PROCEEDINGS

\title{
UCIRC: Infrared Cloud Monitor for EUSO-SPB
}

\author{
Leo Allen, Mikhail Rezazadeh, Stephan Meyer, and Angela V. Olinto* \\ Department of Astronomy \& Astrophysics, KICP, EFI, The University of Chicago, USA \\ E-mail: aolinto@uchicago.edu
}

\section{for the JEM-EUSO Collaboration}

\begin{abstract}
Herein we describe the design and implementation of the University of Chicago Infrared Camera (UCIRC) built for monitoring cloud coverage during the EUSO-SPB flight (Extreme Universe Space Observatory on a Super Pressure Balloon). UCIRC uses 2 infrared (IR) cameras one with a bandpass filter centered on $10 \mu$ and one on $12 \mu$ wavelength, to capture images of the cloud cover under EUSO-SPB in two bands spanning the thermal emission peak. The IR images taken every minute allow the determination of the height, temperature, and density of clouds between the telescope and the ground. We discuss the design and construction of UCIRC, explicating in particular the techniques and design principles that helped make the module temperature and vacuum resilient. Additionally, we outline a pixel by pixel temperature calibration procedure. This paper posits design and implementation methods for cloud cover observation modules on future ultra-high energy space telescopes.
\end{abstract}

35th International Cosmic Ray Conference - ICRC2017

10-20 July, 2017

Bexco, Busan, Korea

${ }^{*}$ Corresponding Author 


\section{Introduction}

Ultra High Energy Cosmic Rays (UHECRs), cosmic rays with energy above $10^{18} \mathrm{eV}$, are currently detected using both Cherenkov water tanks and up-looking fluorescence detectors in giant observatories like the Pierre Auger Observatory [1] in Argentina and the Telescope Array [2] in Utah. UHECR events are detected by means of observing a characteristic particle shower, called an Extensive Air-Shower (EAS), that occurs when a UHECR interacts with Earth's atmosphere. An EAS can be detected by observing atmospheric fluorescence of nitrogen molecules in the 300-400 $\mathrm{nm}$ spectral band. Extreme-Energy Cosmic Rays (EECRs), UHECRs with energy exceeding $5^{19}$ $\mathrm{eV}$, are rare, reaching fluxes of less than 1 per $\mathrm{km}^{2}$ each century. The JEM-EUSO [3] program is aimed at significantly increasing the exposure to UHECRs, particularly EECRs, by observing a large volume of the atmosphere from space. Making the first observation of an UHECR by observing fluorescence from above was the goal of the EUSO-SPB project (the Extreme Universe Space Observatory on a Super Pressure Balloon)[4].

EUSO-SPB carried a 2304 pixel ultraviolet photo-detection module, and a 2 Fresnel lens optical system covering a field of view (FoV) of \pm 6 degrees. The UHECR detector system observed the ground, looking down on EASs rather than up at them. The Photo Detection Module (PDM) on EUSO-SPB was so photosensitive that it only collected data on dark, moonless nights.

The presence of high clouds within the detector FoV can significantly reduce the UHECR event detection rate and event energy calibration. This is because it is possible for the EAS peak signal to occur underneath high clouds. To determine the exposure of EUSO-SPB to UHECRs, it is necessary to determine if some portion of the FoV was precluded from observing EAS by clouds. EUSO-SPB required simultaneous information in location and time about cloud coverage and heights in the part of the atmosphere being observed. This was the responsibility of the University of Chicago Infrared Camera (UCIRC).

\section{Method}

When EUSO-SPB was in observing (night) mode, IR Images of the environmental conditions in and around the effective UHECR detection area were captured by UCIRC every 60 seconds. These images are to be used to collect information about the Cloud Top Heights (CTH) of clouds in the FoV of the UHECR detector. Theses images make it possible to determine the actual exposure of EUSO-SPB to UHECRs by observing whether or not there were clouds in between EUSO-SPB and the ground, and determining the CTHs of all observed clouds.

CTH can be inferred from cloud color temperature (CCT), which can be found using 2 brightness temperatures in bands near the thermal blackbody peak observed by 2 bandpass filtered IR cameras. Typically, a calibrated image in a single band can determine the temperature of a pictured object with a known emissivity, but not when the object has an emissivity coefficient of significantly less than 1 . The clouds below EUSO-SPB have variable emissivity and temperature, but are in front of an ocean of known surface temperature. For a single layer of clouds, the temperature and emissivity of the clouds can be determined with the 2 bands and used to estimate the altitude of the clouds and their optical thickness to UV radiation, improving the UHECR event detection and calibration. Methods for reconstructing CTH using brightness temperatures captured at adjacent 
IR bands are discussed extensively in [5]. The basic principle that motivated our design is that the ratio between the observed brightness temperatures is directly related to the CCT.

\section{Design}

\subsection{IR Camera Implementation}

UCIRC was outfitted with 2 ITAR exempt FLIR Tau 2 IR cameras with $19 \mathrm{~mm}$ lenses. The cameras were focused at infinity because the clouds were expected to be at least 10 kilometers below the payload. The cameras had a field of view of 32 by 24 degrees. The IR cameras had a significantly larger effective field of view than EUSO-SPB. UCIRC takes a pair of pictures every minute that the payload is in "night mode". The wide field of view of the IR cameras makes it possible to extrapolate the cloud conditions in the section of the atmosphere swept out by the PDM field of view during the time between pictures.

The native spectral response of the FLIR cameras was 7.5 to 13.5 microns. One camera was fitted with a SPECTROGON bandpass light filter with a band at wavelengths between 11.5 and 12.9 microns (denoted $12 \mu$ ). The other camera was fitted with an Edmund Optics bandpass light filter that filters out light outside a wavelength band between 9.6 and 11.6 microns (denoted $10 \mu$ ). These bands were spaced so as to get brightness temperature data that facilitates the $\mathrm{CTH}$ reconstruction methods described in [5].

The cameras were outfitted with a Workswell USB3 Module Package that communicates with a host computer, the UCIRC CPU, through a USB3 connection. The UCIRC flight CPU was a Minnowboard Turbot, running UBUNTU 14.04. The CPU had only one USB3 port, so it communicated with the 2 cameras via a 2 port USB3 hub. The power connections running in the USB were disconnected so that the cameras and the hub were powered independently of the CPU (see figure 1).

\subsection{Software}

The Workswell USB3 Module software package is a driver that sits atop a lower level Pleora EBUS driver. It is a suite of high-level commands that can be used to generate IR images from IR camera output. The default image format is JPEG, which is a lossy compressed format. In order to save lossless images, the camera software was redesigned so that the raw ADC output from each pixel was saved individually. The decimal ADC output from a single pixel of a single image is 14 bits, but we added together 4 exposures to generate 16 bit pixel data for 327680 pixels for each image. Taking multiple exposures improved the data signal-to-noise ratio. The images were compressed on the UCIRC CPU using bzip2 to produce $221 \mathrm{kB}$ image files. This lossless compression facilitated efficient data transfer and storage.

Having the cameras capture images simultaneously was of principle importance when we designed the imaging software. The dual core Atom processor on the Minnowboard could process 2 threads concurrently, so, because the cameras could store one image locally, the data transfer time overhead is small. Using one thread for each camera allowed for nearly simultaneous image capture. The average time difference between images captured by the $10 \mu$ camera and the $12 \mu$ camera was just $10 \mathrm{~ms}$. 
Each camera was powered by a $24 \mathrm{~V}$ to $5 \mathrm{~V}$ DC-DC converter that could be switched off using the GPIO pins of the Minnow Board. Periodically power cycling the cameras allowed them to reconnect to the 2 port USB hub which connected them to the CPU. This was carried out using GPIO pin commands from the CPU. The GPIO signal switched a pair of Potter Brumsfeld latching relays (that were driven by a transistor current amplifier) to direct ON/OFF power signals to the cameras.

\subsection{Environment Control}

UCIRC was designed to operate between 0 and $33 \mathrm{~km}$ above sea level during daytime and nighttime. A central design concern was temperature management, specifically with respect to keeping the camera environment at a stable temperature throughout a data collection run. At the 33 kilometer altitude, the IR cameras were subjected extreme temperature shifts between daytime and nighttime. UCIRC had a cubic frame built from 1100 series aluminum panels that were $2.28 \mathrm{~mm}$ thick and $300 \mathrm{~mm}$ long (per side) that were coated with a high emissivity flat white paint. Inside the frame, the components of UCIRC were thermally insulated by FOAMULAR open cell insulation foam.

To maintain a constant operating temperature for the cameras both day and night, an active temperature management system with heaters, Peltier coolers, and heat pipes was used. The active heating and cooling system was controlled by a 2 channel Meerstetter Engineering HV-1123 thermoelectric cooling and heating controller (TEC). Channel 1 of the TEC drives current into 4 Laird Technologies 56995-501 $30 \mathrm{~mm}$ x 30mm Peltier coolers electrically connected in parallel-series. The second channel of the TEC was connected to a $10 \Omega$ Vishay Dale heater resistor. The resistive heater was supposed to be the primary mechanism for heating the camera stage and was to be used in conjunction with the channel 1 Peltier system when heating was needed. The heater resister system was damaged somewhere in transit between Chicago and the Launch Site in New Zealand, so it was not used in flight. The active temperature control system was designed to be more effective at heating than cooling, because in general, UCIRC was actively collecting data during the nighttime when the environment was cold.

The temperature regulated camera stage is a machined aluminum plate, called the main stage, to which both IR cameras (as well as their back end chips) are thermally coupled. This stage is connected with good thermal contact to the Peltier coolers by 4 Enertron sintered powder wick copper heat pipes that lead to the Peltier units at the outside panels. The heat pipes transfer heat pumped by the Peltier units between the camera stage and 4 of the 5 aluminum panel side walls. Machined clamps connected to the ends of the heat pipes transfer heat to and from the Peltiers to the outside panels.

A second thermal stage supports the electronics boards (the CPU, the TEC, the USB hub and the power distribution board). This stage is cooled passively by 3 heat pipes, 1 of which uses methanol as the working fluid while the other 2 use water. The thermal conductivity of the water pipes drops drastically at the freezing point keeping the electronics warm enough to operate at night while providing sufficient cooling during the day.

The TEC-1123-HV uses 4 temperature sensors. It use two NTC thermistors with $5 \mathrm{k} \Omega$ resistance at $20 \mathrm{C}$ measure the sink temperature (one for each channel). The sink temperature is the temperature where 2 of the Peltier coolers contacts the frame panels. The other 2 sensors, Platinum 


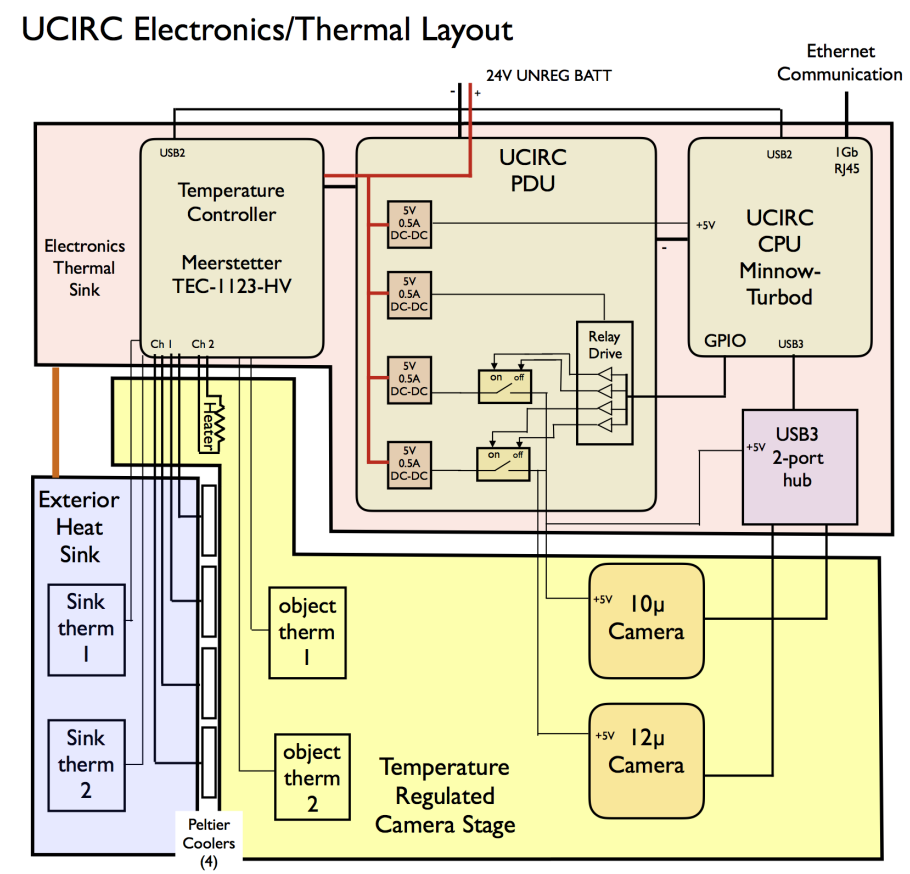

Figure 1: Pictured above is a block diagram of the electronic and thermal components in UCIRC. The 2 cameras communicate with the UCIRC CPU with USB3 using a 2 port hub. The CPU initialized the image capture and handles data management. Images are transferred to the payload computer on a regular basis over ethernet. The cameras are mounted on a temperature stabilized platform controlled with a PID thermal regulator that controls Peltier coolers and a resistive heater. Unregulated $24 \mathrm{~V}$ battery power is converted to $5 \mathrm{~V}$ for the CPU, and power for the cameras can be individually be switched on and off by the CPU. The electronics are passively cooled with heat pipes that lose their thermal conductivity at $0 \mathrm{C}$.

Resistance Thermometers (PRT) operated with a 4 wire readout, measure the object temperature of the center (channel 1) and the edge (channel 2) of the camera thermal stage. The temperature measurement at the center of the main stage controls the TEC Proportional Integral Derivative controller (PID) in order to keep the main stage at a constant, settable temperature. The second sensor is run as a monitor but does not control the PID loop. The parameters of the PID loop were set before flight for stable servo operation and were not changed in flight. The set point temperature for the cameras was set by telemetry command, and was determined by finding the temperature at which the total UCIRC power requirement was minimized for a 24 hour operating cycle at a constant camera temperature.

Because the electronics were to operate near room temperature, the only modification to the electronics boards for flight was to exchange the stock electrolytic power bypass capacitors with ceramic equivalents, designed for vacuum and non vacuum use. The aluminum fins on the Minnowboard's Atom processor were thermally connected to the electronics thermal stage for cooling by $4 \times 100 \mathrm{~mm} 10$ AWG copper wires.

\subsection{Network and Communication}

Infrared camera data is for use in conjunction with the ultraviolet frames collected from EUSO- 
SPB's PDM. For this reason, it is important that the time an infrared image is recorded was synchronized to the same clock that the PDM uses. The MinnowBoard Turbot does not have a high accuracy crystal clock, so there is non-negligible time drift between its CPU time and the CPU time of the main EUSO-SPB payload. To prevent operating system time drift, clock synchronization was achieved by setting up the main payload computer as a Network Time Protocol (NTP) server and slaving UCIRC CPU time to it.

Infrared image data and information about various state parameters for UCIRC was regularly sent from the module to the ground while EUSO-SPB was in flight. This was facilitated by running watchdog programs on the MinnowBoard Turbot. The watchdog programs compress image and parameter data, then transfer files to the main flight computer Secure Copy Protocol (SCP). For most of the flight, an IRIDIUM open port satellite system was used to handle communication and data transfer between EUSO-SPB and ground based systems.

During the course of the flight, commands were sent to the UCIRC system over an Ethernet connection from the main flight PDM computer. These commands originated either internally, as the result of some state on the EUSO-SPB CPU, or externally, as the result of a command being sent over telemetry by an operator on the ground. The most important internal command was the NIGHT command, which indicates that infrared image data is to be being acquired and is based on measurements of the ambient light from an array of photo-transistors. The signal from these photodetectors determines whether or not it is appropriate for the UHECR detector to be turned on, and define the state during which UCIRC was to take infrared images. When signal from the photodetector transistors indicates that data are to be collected, the NIGHT command is sent from the main flight computer to UCIRC, thereby starting a data collection run. When it is determined that external light conditions are inappropriate for UHECR detection, the main flight computer sends the DAY command to UCIRC, ending the IR data collection run.

The data budget for EUSO-SPB was limited by the capabilities of the IRIDIUM system, which transferred about $70 \mathrm{~KB} / \mathrm{s}$. Therefore, an command called INTERVAL was implemented so that operators on the ground could adjust the rate of image capture to ensure that the amount of data collected by UCIRC does not exceed the amount that could be transferred by the telemetry system.

In addition to controlling the rate of image capture, external commands called TEC PARAMETER(s) were sent during the flight to help TEC keep UCIRC temperature stable. Several TEC parameters were available for adjustment by a ground controller, each with their own command. The parameters controlled by these commands included the target temperature of the main stage, the maximum current and voltage allowed through the Peltier coolers, among others.

Each type of command (NIGHT, INTERVAL, and TEC PARAMETER) were sent as text from the main flight computer to the MinnowBoard Turbot via a TCP/IP connection. The MinnowBoard has a static IP address, and is always running a server program that waits for a connection from the main flight computer (which acts as the client). The main flight computer serves as an intermediary between external (telemetry) commands and UCIRC.

\section{Testing and Calibration}

Our system was tested in a thermovac chamber that replicated the expected flight environment. The chamber was pumped down to 0.3 mbar and then the shroud and floor of the chamber 
was cooled with liquid nitrogen. UCIRC and a calibrator unit were loosely thermally coupled to the cooled floor of the chamber. The calibrator unit housed an insulated adjustable temperature blackbody target that almost completely fills the field of view of both cameras (when the calibrator is attached to UCIRC). After confirming that UCIRC would turn on and take pictures in a vacuum environment, we conducted a temperature calibration. The calibrator was taken through a range of temperatures between $-15.0 \mathrm{C}$ and $22.5 \mathrm{C}$. Because of time constraints, the system was not stabilized at each temperature and the calibrator did not reach a completely stable equilibrium at the colder set points in the thermovac chamber. A subsequent calibration run not in the thermovac chamber was carried out on the ground in New Zealand with a calibrator temperature range of $0 \mathrm{C}$ to $30 \mathrm{C}$.
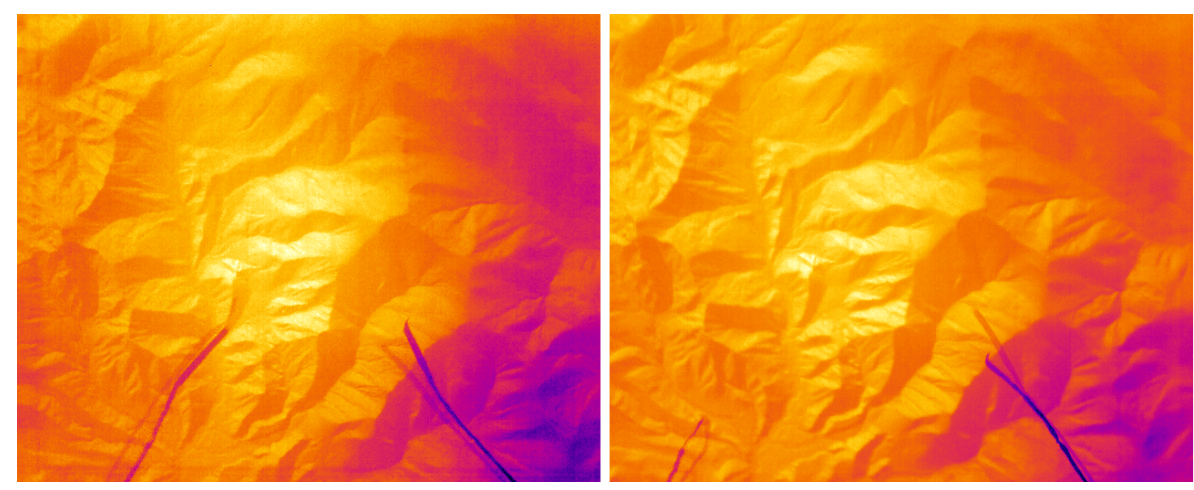

Figure 2: Infrared images of the New Zealand Southern Alps taken by UCIRC while EUSO-SPB ascended. The left image was centered at $12 \mu$, the right image was centered at $10 \mu$. Note that the bottom right of the image is significantly colder than the middle, indicating that the pixels do not have precisely the same power response function.
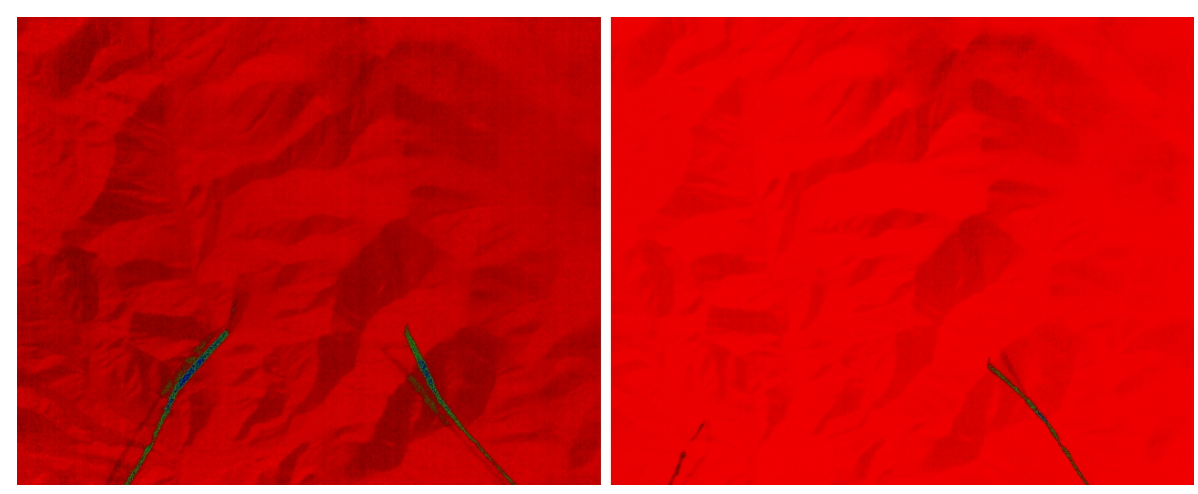

Figure 3: Temperature calibrated images of the Southern Alps taken by UCIRC while EUSO-SPB ascended. The lowest temperature recorded by the $12 \mu$ filtered camera (left) is $251.856 \mathrm{~K}$, while the highest temperature recoded by that camera is $260.159 \mathrm{~K}$. These temperatures are slightly colder than they ought to be, so we are doing further work with the calibration model. Note that the calibration flattens out the low temperature bubble on the bottom right hand corner of each image

First, each IR camera was calibrated pixel by pixel by mapping a set of raw decimal outputs from each pixel to a corresponding set of calibration temperatures using the data from the ther- 
movac test. The calibration temperatures lower bound was set by what was achievable with the cooler in the calibrator, and the upper bound was near room temperature. Each pixel was independently fit to a cubic function to model the set calibrator temperature as a function of observed pixel output. The fit for each pixel converts each raw image into a brightness temperature image for each camera.

The calibration based on data taken in the thermovac did not always convert pixel ADC values to a reasonable temperature. For this reason, UCIRC itself was also taken through a controlled range of temperatures between $15 \mathrm{C}$ and $30 \mathrm{C}$ as part of the calibration data collection done in New Zealand, to get a sense for how the pixel ADC output responds to camera temperature. The calibration data collected in New Zealand indicate that in addition to the temperature of the object being imaged, the camera temperature must be factored in to the calibration model. These data are being worked through now.

\section{Conclusion: Flight Discussion}

UCIRC's temperature control, communication, and imaging systems worked as designed during the ascent, pressurization, and initial float phases of EUSO-SPB's launch. After the initial float phase, the temperature control system failed because a default setting forced the TEC to shut off when the sink temperature (which was essentially the outside temperature) fell below -40C. When this happened, the entire system would freeze up at night so IR data were not collected. This setting was fixed using SSH a few days into the flight, but UCIRC was de facto nonfunctional the entire time the temperature control system was down.

During the time that UCIRC was functional, there were still data acquisition issues caused by the workswell image capture drivers. The precise nature of the issue with the capture drivers was not determined, but the tangible effect was that there are frequent holes in time for IR data.

The issues that precluded UCIRC from taking data for much of the flight do not necessarily indicate that a similar system would be unsuited for observing atmospheric conditions below a similar EUSO payload.

\section{References}

[1] A. Aab et al., (Pierre Auger Collaboration), The Pierre Auger Cosmic Ray Observatory, Nuclear Instruments and Methods in Physics Research A (798) 172-213 (2015).

[2] T. Abu-Zayyad, et al. (Telescope Array Collaboration), The Surface Detector Array of the Telescope Array Experiment, Nuclear Instruments and Methods in Physics Research A (689) 87-97 (2012).

[3] J. H. Adams Jr. et al., (JEM-EUSO Collaboration), The JEM-EUSO mission: An introduction, Experimental Astronomy 40 3-17 (2015).

[4] L. Wiencke for the JEM-EUSO Collaboration EUSO-SPB Mission and Science, ICRC 2017. These Proceedings

[5] I. Tabone et al., Comparing different methods to retrieve cloud top height from Meteosat satellite data., SPIE Remote Sensing of Clouds and the Atmosphere, Toulouse France, 2015. 